\title{
Dispersión de restos de disparos de armas de fuego en blancos cercanos, con microscopio electrónico de barrido (MEB)
}

\author{
Rosa Carrera, Shérmany Aronés, Alcides López, Marco Villacorta \\ Instituto de Medicina Legal, Ministerio Público, e Instituto de Patología, UNMSM
}

Introducción: Se ha estudiado la dispersión de restos de pólvora de armas de fuego disparados a distancias cortas sobre hojas bond blancas, por medio de análisis morfológico macroscópico directo, análisis morfológico por microscopia electrónica de barrido, mediante electrones secundarios, y de contraste Z mediante electrones retrodispersados. Esta microscopia ha servido también para hacer análisis multielemental por medio de espectroscopia de rayos $X$ característicos.

Objetivos: Establecer dispersión de restos de disparos por armas de fuego en blancos cercanos.

Diseño: Experimental.

Institución: Instituto de Medicina Legal, Ministerio Público, e Instituto de Patología, UNMSM.

Materiales: Disparos experimentales sobre blancos de papel a distancias conocidas.

Intervenciones: Restos de pólvora con disparos a 0, 2, 15 y $50 \mathrm{~cm}$ de distancia.

Principales medidas de resultados: Medidas de dispersión.

Resultados: Obteniéndose mapeos elementales de diferentes zonas próximas al orificio de ingreso, los análisis mostraron presencia de elementos químicos $\mathrm{Pb}, \mathrm{Sb}$ y Ba entre los materiales propios del arma de fuego; de acuerdo a lo esperado, se encontró patrón de dispersión de partículas alrededor del orifico de entrada, que depende de la distancia del disparo. Se analizó restos del disparo en orificio cutáneo.

Conclusiones: Se encontró concordancia con lo obtenido con los métodos alternativos.

Palabras clave: Microscopia electrónica de barrido, distancia de disparo, restos de disparo.

\section{Evaluación de los antígenos totales de Fasciola hepatica como agente de absorción de anticuerpos inespecíficos en el inmunodiagnóstico de la toxocarosis humana}

Yrma Espinoza, William Roldán, Herman Vildósola, Susana Jiménez Instituto de Medicina Tropical Daniel A. Carrión, Facultad de Medicina, UNMSM

Objetivos: Evaluar la capacidad de absorción de anticuerpos inespecíficos de antígenos totales de adultos de Fasciola hepatica frente a los de Ascaris suum, para mejorar el serodiagnóstico de toxocarosis humana. Diseño: Observacional, descriptivo

Institución: Instituto de Medicina Tropical Daniel A. Carrión, Facultad de Medicina, UNMSM.

Participantes: Se recolectó sueros de pacientes con toxocarosis, otras helmintiasis y de personas aparentemente sanas. Se preparó antígenos TES de Toxocara canis, de Fasciola hepatica y de Ascaris suum.

Intervenciones: SDS-PAGE y western blot.

Principales medidas de resultados: Los antígenos fueron analizados por SDS-PAGE y western blot y la capacidad de absorción de anticuerpos inespecíficos mediante ELISA y western blot.

Resultados: Por SDS-PAGE y western blot se determinó que el extracto antigénico de $F$. hepatica posee bandas antigénicas $8,12,17,23,27,30,36,43,66$ y $136 \mathrm{KDa}$ y que logra reducir las reacciones cruzadas en forma similar a los antígenos de $A$. suum. Mediante western blot se observó que el antígeno de $67 \mathrm{KDa}$ de los antígenos TES posee cierta reactividad cruzada con alguno de los componentes del extracto antigénico de $F$. hepatica.

Conclusiones: El extracto antigénico de F. hepatica puede ser utilizado para la absorción de anticuerpos inespecíficos en el inmunodiagnóstico de toxocarosis humana.

Palabras clave: Antígenos, Fasciola hepatica, anticuerpos, diagnóstico, toxocarosis. 